\title{
Persistent clotting protein pathology in Long COVID/Post-Acute Sequelae of COVID-19 (PASC) is accompanied by increased levels of antiplasmin
}

Etheresia Pretorius $^{1 *} \mathbb{0}$, Mare Vlok² ${ }^{2}$ Chantelle Venter ${ }^{1}$, Johannes A. Bezuidenhout ${ }^{1}$, Gert Jacobus Laubscher ${ }^{3}$, Janami Steenkamp ${ }^{1,4}$ and Douglas B. Kell ${ }^{1,5,6^{*}}$ (D)

\begin{abstract}
Background: Severe acute respiratory syndrome coronavirus 2 (SARS-Cov-2)-induced infection, the cause of coronavirus disease 2019 (COVID-19), is characterized by acute clinical pathologies, including various coagulopathies that may be accompanied by hypercoagulation and platelet hyperactivation. Recently, a new COVID-19 phenotype has been noted in patients after they have ostensibly recovered from acute COVID-19 symptoms. This new syndrome is commonly termed Long COVID/Post-Acute Sequelae of COVID-19 (PASC). Here we refer to it as Long COVID/PASC. Lingering symptoms persist for as much as 6 months (or longer) after acute infection, where COVID-19 survivors complain of recurring fatigue or muscle weakness, being out of breath, sleep difficulties, and anxiety or depression. Given that blood clots can block microcapillaries and thereby inhibit oxygen exchange, we here investigate if the lingering symptoms that individuals with Long COVID/PASC manifest might be due to the presence of persistent circulating plasma microclots that are resistant to fibrinolysis.
\end{abstract}

Methods: We use techniques including proteomics and fluorescence microscopy to study plasma samples from healthy individuals, individuals with Type 2 Diabetes Mellitus (T2DM), with acute COVID-19, and those with Long COVID/PASC symptoms.

Results: We show that plasma samples from Long COVID/PASC still contain large anomalous (amyloid) deposits (microclots). We also show that these microclots in both acute COVID-19 and Long COVID/PASC plasma samples are resistant to fibrinolysis (compared to plasma from controls and T2DM), even after trypsinisation. After a second trypsinization, the persistent pellet deposits (microclots) were solubilized. We detected various inflammatory molecules that are substantially increased in both the supernatant and trapped in the solubilized pellet deposits of acute COVID19 and Long COVID/PASC, versus the equivalent volume of fully digested fluid of the control samples and T2DM. Of particular interest was a substantial increase in a(2)-antiplasmin (a2AP), various fibrinogen chains, as well as Serum Amyloid A (SAA) that were trapped in the solubilized fibrinolytic-resistant pellet deposits.

\footnotetext{
*Correspondence: resiap@sun.ac.za; dbk@liv.ac.uk

${ }^{1}$ Department of Physiological Sciences, Faculty of Science, Stellenbosch

University, Private Bag X1 Matieland, Stellenbosch 7602, South Africa

${ }^{5}$ Department of Biochemistry and Systems Biology, Institute of Systems,

Molecular and Integrative Biology, Faculty of Health and Life Sciences,

University of Liverpool, Liverpool L69 7ZB, UK

Full list of author information is available at the end of the article
}

(c) The Author(s) 2021. Open Access This article is licensed under a Creative Commons Attribution 4.0 International License, which permits use, sharing, adaptation, distribution and reproduction in any medium or format, as long as you give appropriate credit to the original author(s) and the source, provide a link to the Creative Commons licence, and indicate if changes were made. The images or other third party material in this article are included in the article's Creative Commons licence, unless indicated otherwise in a credit line to the material. If material is not included in the article's Creative Commons licence and your intended use is not permitted by statutory regulation or exceeds the permitted use, you will need to obtain permission directly from the copyright holder. To view a copy of this licence, visit http://creativecommons.org/licenses/by/4.0/. The Creative Commons Public Domain Dedication waiver (http://creativeco mmons.org/publicdomain/zero/1.0/) applies to the data made available in this article, unless otherwise stated in a credit line to the data. 
Conclusions: Clotting pathologies in both acute COVID-19 infection and in Long COVID/PASC might benefit from following a regime of continued anticlotting therapy to support the fibrinolytic system function.

Keywords: COVID-19, Long COVID/PASC, Fibrin(ogen), Microclots, Proteomics, Antiplasmin, Serum Amyloid A

\section{Introduction}

Severe acute respiratory syndrome coronavirus 2 (SARSCov-2)-induced infection, the cause of coronavirus disease 2019 (COVID-19), is characterized by acute clinical pathologies, including various coagulopathies that may result in either bleeding and thrombocytopenia, hypercoagulation, pulmonary intravascular coagulation, microangiopathy venous thromboembolism or arterial thrombosis [1-9]. Acute COVID-19 infection is also characterized by dysregulated, circulating inflammatory biomarkers, hyperactivated platelets, damaged erythrocytes and substantial deposition of microclots in the lungs $[6,8-16]$. Acute COVID-19 patients may suffer from thrombocytopenia that may lead to life-threatening disseminated intravascular coagulation (DIC) [17]. Predisposing risk factors or co-morbidities that may also lead to a poor prognosis of acute COVID-19, are cardiovascular disease, diabetes, arterial hypertension, obesity $[3,8,9,18-21]$, as well as cancer [22]. Complications like liver injury, acute respiratory distress syndrome (ARDS), sepsis, myocardial injury, renal insufficiency and Multiple Organ Dysfunction Syndrome (MODS) are common in cancer patients with COVID-19 [22]. Plasma of COVID19 patients also carries a significant load of preformed amyloid clots [6] and this phenomenon may be indicative of a poor prognosis.

Recently, a new COVID-19 phenotype has been noted in patients after they have ostensibly recovered from acute COVID-19 symptoms. This new syndrome is commonly termed Long COVID/Post-Acute Sequelae of COVID-19 (PASC) [23]. We use the terminology Long COVID/PASC. Long COVID/PASC can involve sequelae and other medical complications that last for weeks to months after initial recovery, and may include more than 50 long-term effects [24]. Preliminary data about Long COVID/PASC symptoms show numerous similarities to chronic illnesses [23, 25-27] known to be associated with viral infections, such as Myalgic Encephalomyelitis/ Chronic Fatigue Syndrome (ME/CFS) [28, 29], postural orthostatic tachycardia syndrome[30] and Mast Cell Activation Syndrome [31, 32]. Mast cell activation has also been suggested to play an important role in Long COVID/PASC, due to atypical responses to SARS-CoV-2 by the dysfunctional mast cells [31].

Lingering symptoms have been found to persist for as much as 6 months (or longer) after acute infection, where COVID-19 survivors complain of recurring fatigue or muscle weakness, being out of breath, sleep difficulties, and anxiety or depression [32]. Patients who were more severely ill during their hospital stay tended to have more severe impaired pulmonary diffusion capacities and abnormal chest imaging manifestations, and are the main target population for interventions for long-term recovery [32]. However, it was also shown that $\sim 32 \%$ of subjects reporting symptoms at $61+$ days after infection were asymptomatic at the time of initial SARS-CoV-2 testing [32]. Many patients are also developing Long COVID/PASC after mild or asymptomatic infection, despite not being hospitalized [33]. Researchers in Italy found that $87.4 \%$ of 143 COVID-19 patients reported at least one symptom 60 days post-infection, and $55 \%$ had three or more. According to their research [25], the most common symptoms were: fatigue (53.1\%), difficulty in breathing (43.4\%), joint pain $(27.3 \%)$ and chest pain $(21.7 \%)$. In the UK it was found in a study of 384 patients (mean age 59.9 years; 62\% male) followed for a median 54 days post discharge, $53 \%$ reported persistent breathlessness, 34\% cough and 69\% fatigue and $14.6 \%$ had depression [34]. In those discharged with elevated biomarkers, $30.1 \%$ and $9.5 \%$ had persistently elevated D-dimer and $\mathrm{C}$ reactive protein, respectively. $38 \%$ of chest radiographs remained abnormal with $9 \%$ deteriorating [34]. In the largest global study to-date on this issue, a survey of 3,762 Long COVID/PASC patients, from 56 countries found nearly half still could not work full-time 6 months post-infection, due mainly to fatigue, post-exertional malaise, and cognitive dysfunction [35].

In the current study, we investigate if we could find dysregulated molecules in circulation that might cause the lingering symptoms in individuals with Long COVID/ PASC. These symptoms might be due to the presence of persistent circulating plasma clots that are resistant to fibrinolysis. We have previously shown that large anomalous (amyloid) fibrin(ogen) deposits are present in plasma from acute COVID-19 patients [8, 9]. Here we show that plasma samples from Long COVID/PASC still contain large anomalous (amyloid) deposits, and that these deposits are most resistant to fibrinolysis, even with the treatment of a two-step trypsin method. We used proteomics to study the protein presence in both digested supernatant and trapped persistent pellet deposits (after protein digestion via trypsin). Of particular interest was a substantial increase in the acute phase inflammatory molecule Serum Amyloid A (SAA4) and $\alpha(2)$-antiplasmin 
$(\alpha 2 \mathrm{AP})$ that were trapped in the fibrinolytic-resistant pellet deposit. The plasmin-antiplasmin system plays a key role in blood coagulation and fibrinolysis [36]. Plasmin and $\alpha 2 \mathrm{AP}$ are primarily responsible for a controlled and regulated dissolution of the fibrin polymers into soluble fragments [36, 37].

\section{Materials and methods Ethical clearance}

Ethical clearance for the study was obtained from the Health Research Ethics Committee(HREC) of Stellenbosch University (South Africa) (reference: N19/03/043, project ID: 9521). The experimental objectives, risks, and details were explained to volunteers and informed consent were obtained prior to blood collection. Strict compliance to ethical guidelines and principles Declaration of Helsinki, South African Guidelines for Good Clinical Practice, and Medical Research Council Ethical Guidelines for Research were kept for the duration of the study and for all research protocols.

\section{Sample demographics and considerations}

Blood was collected from healthy volunteers $(\mathrm{N}=13 ; 6$ males, 7 females; mean age: $52.4 \pm 4.8$ ) to serve as controls. Healthy volunteers did not smoke, or suffer from cardiovascular diseases or coagulopathies and pregnancy, lactation, and the use of anticoagulants, were exclusion criteria [38]. Patients diagnosed with COVID-19 (before treatment) $(\mathrm{N}=15 ; 9$ males and 6 females; mean age: $54.8 \pm 15.3)$ and also patients with Type 2 Diabetes Mellitus (T2DM) $(\mathrm{N}=10$; 7 males and 3 females; mean age: $59.2 \pm 15.9)$ were included in this study. In addition, patients that suffered from Long COVID/PASC $(\mathrm{N}=11 ; 3$ males and 8 females; mean age: $55.7 \pm 5.8)$, were included. Of these patients, three were classified with severe acute COVID-19 symptoms and they were hospitalized where they received oxygen. One person was classified as severe and on ventilation. Two patients were classified as having moderate COVID-19 symptoms, with hospitalization and oxygen. One patient was diagnosed with moderate symptoms but was not hospitalized. Four patients presented with mild acute COVID19 symptoms and were not hospitalized. These patients suffered from persistent Long COVID/PASC symptoms for at least 2 months after they have recovered from acute COVID-19. None of the Long COVID/PASC individuals were diagnosed with T2DM before their acute COVID19 diagnosis.

\section{Blood sample collection}

Either a qualified phlebotomist or medical practitioner drew the citrated blood samples [4.5 mL sodium citrate (3.2\%) tubes (BD Vacutainer $\left.\left.{ }^{\circledR}, 369714\right)\right]$, via venepuncture, adhering to standard sterile protocol. Whole blood (WB) was centrifuged at $3000 \times g$ for $15 \mathrm{~min}$ at room temperature and the supernatant platelet poor plasma (PPP) samples were collected and stored in $1.5 \mathrm{~mL}$ Eppendorf tubes at $-80^{\circ} \mathrm{C}$.

\section{Viscometry}

Plasma viscosity was measured with the microVISC $^{\mathrm{TM}}$ viscometer (RheoSense Inc., CA United States), which uses Viscometer/Rheometer On-a-Chip (VROC ${ }^{\circledR}$ ) microfluidic sensor technology. Prior to analysis, stored PPP aliquots from the samples were thawed from -80 to $35{ }^{\circ} \mathrm{C}$ with the use of an incubator. The samples were also kept at $35{ }^{\circ} \mathrm{C}$ in the incubator during measurements to keep the temperature constant, as it one of the variables used to measure viscosity. Between each measurement, the micro-viscometer was cleaned with $1 \%$ Scienceware ${ }^{\circledR}$ Aquet $^{\circledR}$ liquid detergent solution (Sigma-Aldrich, Z273260), in order to maintain stable viscosity measurements. Plasma viscosity was calculated according to Newton's law of viscosity:

$$
\mu=\frac{\tau}{\Upsilon_{a p p}}
$$

where $\mu$ is the viscosity, $\tau$ is the shear stress, and $\Upsilon_{\text {app }}$ is the apparent shear rate.

\section{Serum Amyloid A ELISA analysis}

ELISA analysis was performed on PPP from 12 controls, 11 individuals with acute COVID-19 and 11 individuals with Long COVID/PASC. The Human SAA ELISA Kit (SAA1) (E-EL-H2183, Elabscience Biotechnology Inc.) was used in this analysis following manufacturer prescribed protocol. SAA1 is one of the two acute phase SAA proteins [39].

\section{Platelet pathology}

The whole blood (WB) (haematocrit) samples of healthy volunteers, COVID-19 and Long COVID/PASC patients were exposed to the two fluorescent markers, CD62P (PE-conjugated) (platelet surface P-selectin) (IM1759U, Beckman Coulter, Brea, CA, USA) and PAC-1 (FITC-conjugated) (340507, BD Biosciences, San Jose, CA, USA). CD62P is found in the granules of platelets and then translocate to the platelet membrane surface. The translocation occurs after the platelet P-selectin is released from the cellular granules during platelet activation $[6,9]$. $4 \mu \mathrm{L} \mathrm{CD62P}$ and PAC-1 was added to $20 \mu \mathrm{L}$ haematocrit. The haematocrit exposed to the markers was incubated for $30 \mathrm{~min}$ (protected from light) at room temperature. The excitation wavelength for PAC-1 was set at 450 to $488 \mathrm{~nm}$ and the emission at 499 to $529 \mathrm{~nm}$ and for the CD62P marker it was $540 \mathrm{~nm}$ to $570 \mathrm{~nm}$ and the emission 
$577 \mathrm{~nm}$ to $607 \mathrm{~nm}$. Processed samples were viewed using the Zeiss Axio Observer 7 fluorescent microscope with a Plan-Apochromat $63 \times / 1.4$ Oil DIC M27 objective (Carl Zeiss Microscopy, Munich, Germany).

Platelet poor plasma (PPP): Amyloid (fibrin(ogen) protein and anomalous clotting in platelet poor plasma samples, before and after two trypsin digestion protocols

All naïve PPP samples from T2DM, controls, acute COVID-19 and Long COVID/PASC PPP were studied using florescence microscopy. For the proteomics analysis 5 healthy, 4 Long COVID/PASC and 9 acute COVID19 samples were used (one acute COVID-19 donor's repeat sample, taken 2 days after the first donation were included). PPP samples were prepared for proteomics. One of the Long COVID/PASC volunteers previously donated a (healthy) blood sample. Here we also compare his healthy and Long COVID/PASC samples before and after trypsin digestion.

\section{Naïve PPP samples: fluorescence microscopy to show anomalous clotting and microclot formation}

To study anomalous clotting of fibrin(ogen) and plasma proteins, in naïve PPP samples PPP (healthy PPP, T2DM, COVID-19, Long COVID/PASC) were exposed to the fluorescent amyloid dye, Thioflavin $\mathrm{T}$ (ThT) (final concentration: 0,005 mM) (Sigma-Aldrich, St. Louis, MO, USA) for $30 \mathrm{~min}$ at room temperature. This ThT method was developed to visualize anomalous clotting in inflammatory conditions [40-43]. After incubation, $4 \mathrm{uL}$ PPP and placed on a glass slide and covered with a coverslip. The excitation wavelength for ThT was set at $450 \mathrm{~nm}$ to $488 \mathrm{~nm}$ and the emission at $499 \mathrm{~nm}$ to $529 \mathrm{~nm}$ and processed samples were viewed using a Zeiss Axio Observer 7 fluorescent microscope with a Plan-Apochromat $63 \times / 1.4$ Oil DIC M27 objective (Carl Zeiss Microscopy, Munich, Germany) [5, 8, 9].

\section{Two trypsin digestions protocols of platelet poor plasma (PPP)}

Chemicals used for proteomics The following reagents were purchased from Sigma: ammonium bicarbonate, ammonium acetate, Sodium Chloride $(\mathrm{NaCl})$, Tris, sodium duodecucyl sulphate (SDS), Triscarboxyethylphosphine (TCEP), methane methylthioslfonate, (MMTS), Trifluoroacetic acid (TFA) and $3 \mathrm{M}$ Empore C18 solid phase extraction discs. Trypsin was purchased from New England Biosystems and HILIC MagResyn functionalised particles from ReSynBiosciences. Acetonitrile was purchased from Romil and Chloroform was obtained from Merck.

Two trypsin digestion protocols were followed (see Fig. 1). It is well-known that trypsin digests protein, however, we adapted the trypsin digestion step for a first digestion step for this experiment. The non-denaturing digest (1 Trypsin method) is our own method. The second digestion step was done according to https://resynbio.com/wp-content/uploads/2019/12/ HILIC_RAPOBD.pdf. With the following modifications: DTT was replaced with TCEP and IAA with MMTS, The trypsin concentration was was lowered as the amount of protein wasn't known and the incubation time extended to $18 \mathrm{~h}$.

The first trypsin digestion protocol was followed using PPP from 5 healthy, 4 Long COVID/PASC and 9 acute COVID volunteers (one acute COVID-19 donor's repeat sample, taken 2 days after the first donation was included). The supernatant proteomics data was collected from these samples. The trypsin digestion was also done with 4 volunteers with T2DM. Here we also included a repeat sample of one of the volunteers to confirm results. The second-step trypsinization protocol was performed on the undigested pellet deposits from 5 acute COVID-19 samples and 4 Long COVID/ PASC samples. This second-step trypsinization was also performed on 5 control plasma samples.

First trypsin digestion protocol of nä̈ve PPP All PPP samples were exposed to a first step of trypsin digestion of the plasma proteins. The samples were diluted in ten times in $10 \mathrm{mM}$ ammonium bicarbonate and protein concentration determined. The samples were standardised to $1 \mathrm{mg} / \mathrm{mL}$ total protein. A total of $1 \mu \mathrm{g}$ trypsin (New England Biosystems) was added to the plasma for 1:50 enzyme to substrate ratio. No reduction or alkylation was performed. After this first trypsin digestion protocol, both COVID-19 and Long COVID/PASC samples formed a visible pellet deposit at the bottom of the tubes after centrifugation for $30 \mathrm{~min}$ at $13,000 \times \mathrm{g}$. Healthy PPP samples and the T2DM PPP samples did not form any visible deposit at the bottom of the tube.

Fluorescence microscopy of trypsin-degraded supernatant and visible pellet deposits After the first trypsin digestion protocol, supernatants were removed and the remaining $10 \mu \mathrm{L}$ was exposed to ThT (as previously described) and the rest of the supernatants were analysed using mass spectrometry (seen methods below). The $10 \mu \mathrm{L}$ of healthy PPP and T2DM PPP contained no pellet deposit, while the $10 \mu \mathrm{L}$ of the COVID-19 and Long COVID/PASC sample did contain a visible pellet deposit. These samples were also visualized using the Zeiss Axio Observer 7 fluorescent microscope with a Plan-Apochromat 63×/1.4 Oil DIC M27 objective (Carl Zeiss Microscopy, Munich, Germany). 


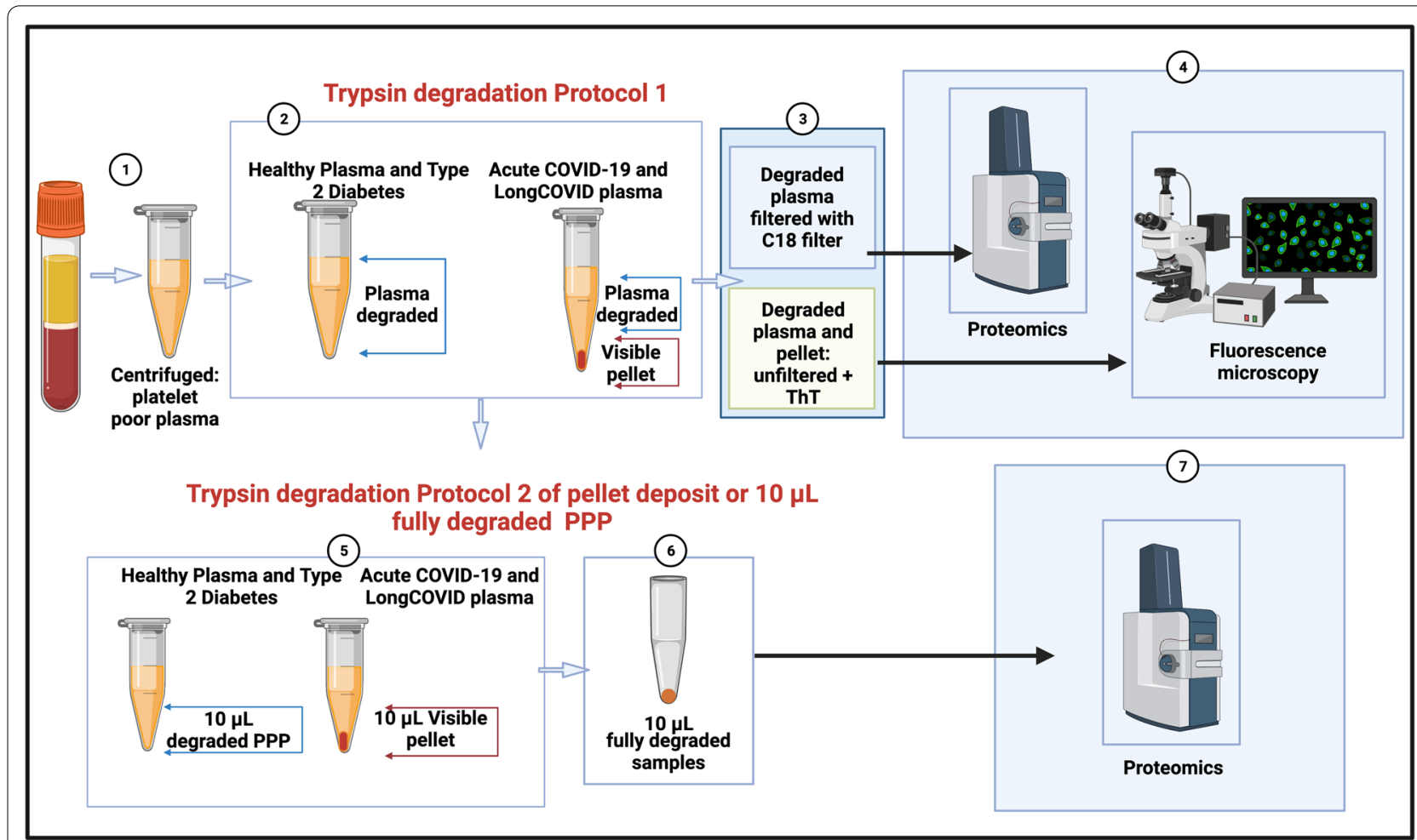

Fig. 1 Two trypsin digestion protocols, followed by fluorescence microscopy and proteomics of platelet poor plasma (PPP) from healthy individuals, patients with Type 2 Diabetes Mellitus (T2DM), COVID-19 and Long COVID/PASC. (1) Citrated blood was centrifuged to obtain PPP. (2) PPP were treated with trypsin to allow plasma protein digestion. Health PPP and T2DM PPP were fully degraded. COVID-19 and Long COVID/PASC sample formed a undigested pellet deposit at the bottom of the tubes. ( 3 and $\mathbf{4})$ For fluorescence microscopy, the supernatants were removed and the remaining $10 \mu \mathrm{L}$ of supernatant and/or pellet samples were exposed to thioflavin $\mathrm{T}$ (ThT) and viewed with fluorescence microscope. Before liquid chromatography-mass spectrometry (LC-MS) based proteomics, supernatants were passed through a $\mathrm{C}_{18}$ solid phase extraction (SPE) device. (5) A second trypsin digestion protocol was followed to (6) degrade the pellet deposit in the COVID-19 and Long COVID/PASC samples. The same method was followed with healthy and T2DM PPP (although these samples did not contain a visible pellet deposit). (7) Double-trypsinized samples from controls, COVID-19 and Long COVID/PASC samples were then studied using proteomics. (Figure created with BioRender.com)

\section{Pellet deposit digestion by a second trypsin digestion protocol}

$60 \mu \mathrm{L}$ of chloroform was added to the remaining $10 \mu \mathrm{L}$ PPP, containing the pellet deposit and again centrifuged at $13,000 \times g$ for $30 \mathrm{~min} .50 \mu \mathrm{L}$ supernatant was removed and the rest airdried. This deposit was further dissolved in $100 \mathrm{mM}$ Tris ( $\mathrm{pH}$ 8.5) containing 1\% (sodium dodecyl sulphate (SDS) (Sigma) and $5 \mathrm{mM}$ (tris(2-carboxyethyl)phosphine) (TCEP, Sigma), and reduced at $45{ }^{\circ} \mathrm{C}$ for $1 \mathrm{~h}$. The product was cooled to room temperature and Cysteine residues blocked withmethyl methanethiosulfonate (MMTS, Sigma). These samples were also studied using mass spectrometry. The reduced and thiomethylated protein samples were diluted 1:1 with $200 \mathrm{mM}$ ammonium acetate (Sigma) containing 30\% acetonitrile (ACN, Romil), $\mathrm{pH}$ 4.5. The samples were incubated with HILIC functionalised magnetic particles (ResynBiosciences) equilibrated with $100 \mathrm{mM}$ ammonium acetate containing $15 \% \mathrm{ACN}, \mathrm{pH} 4.5$ for
$30 \mathrm{~min}$. After, binding, the supernatant was removed and the particles washed twice with $95 \%$ ACN. To each sample $0.1 \mathrm{mg}$ of trypsin was added in $10 \mathrm{mM}$ ammonium bicarbonate. The samples were incubated over night at $37{ }^{\circ} \mathrm{C}$ with agitation. After $18 \mathrm{~h}$ the supernatant was removed and the particles washed with $1 \%$ trifluoroacetic acid (TFA, Sigma). The wash was combined with the first supernatant and applied to a $\mathrm{C}_{18}$ SPE (3 M Emopore) device prior to analysis.

\section{Proteomics of platelet poor plasma}

After the first trypsin digestion protocol, the supernatants were subjected to $\mathrm{C}_{18}$ and solid phase extraction (SPE) and proteomics were performed. After the pellet deposit was solubilized in the second trypsin digestion protocol, the now soluble pellet deposits were also studied using proteomics. 


\section{Liquid chromatography on degraded supernatant} and degraded pellet deposit

Dionex nano-RSLC Liquid chromatography was performed on a Thermo Scientific Ultimate 3000 RSLC [44] equipped with a $20 \mathrm{~mm} \times 100 \mu \mathrm{m} \mathrm{C}_{18}$ trap column (Thermo Scientific) and a CSH $25 \mathrm{~cm} \times 75 \mu \mathrm{m} 1.7 \mu \mathrm{m}$ particle size $\mathrm{C}_{18}$ column (Waters) analytical column. The solvent system employed was loading: $2 \%$ acetonitrile:water; $0.1 \%$ FA; Solvent A: $2 \%$ acetonitrile:water; $0.1 \%$ FA and Solvent B: $100 \%$ acetonitrile:water. The samples were loaded onto the trap column using loading solvent at a flow rate of $2 \mu \mathrm{L} / \mathrm{min}$ from a temperature controlled autosampler set at $7{ }^{\circ} \mathrm{C}$. Loading was performed for $5 \mathrm{~min}$ before the sample was eluted onto the analytical column. Flow rate was set to $300 \mathrm{~nL} / \mathrm{min}$ and the gradient generated as follows: $5.0 \%-30 \% \mathrm{~B}$ over $60 \mathrm{~min}$ and $30-50 \% \mathrm{~B}$ from 60-80 $\mathrm{min}$. Chromatography was performed at $45^{\circ} \mathrm{C}$ and the outflow delivered to the mass spectrometer.

\section{Mass spectrometry of platelet poor plasma}

Mass spectrometry was performed using a Thermo Scientific Fusion mass spectrometer equipped with a Nanospray Flex ionization source. Plasma samples, before and after addition of spike protein addition $\left(1 \mathrm{ng} \mathrm{mL} L^{-1}\right.$ final exposure concentration), from 4 of our control samples were analysed with this method. The sample was introduced through a stainless-steel nano-bore emitter Data was collected in positive mode with spray voltage set to $1.8 \mathrm{kV}$ and ion transfer capillary set to $275{ }^{\circ} \mathrm{C}$. Spectra were internally calibrated using polysiloxane ions at $\mathrm{m} / \mathrm{z}=445.12003$. MS1 scans were performed using the orbitrap detector set at 120,000 resolution over the scan range 375-1500 with AGC target at 4 E5 and maximum injection time of $50 \mathrm{~ms}$. Data was acquired in profile mode.MS2 acquisitions were performed using monoisotopic precursor selection for ion with charges $+2-+7$ with error tolerance set to $\pm 10 \mathrm{ppm}$. Precursor ions were excluded from fragmentation once for a period of $60 \mathrm{~s}$. Precursor ions were selected for fragmentation in HCD mode using the quadrupole mass analyser with HCD energy set to $30 \%$. Fragment ions were detected in the Orbitrap mass analyzer set to 30,000 resolution. The AGC target was set to $5 \mathrm{E} 4$ and the maximum injection time to $100 \mathrm{~ms}$. The data was acquired in centroid mode.

\section{Mass spectrometry data analysis}

The raw files generated by the mass spectrometer were imported into Proteome Discoverer v1.4 (Thermo Scientific) and processed using the Sequest HT algorithm. Database interrogation was performed against the 2019-nCOVpFASTA database. Semi-tryptic cleavage with 2 missed cleavages was allowed for. Precursor mass tolerance was set to $10 \mathrm{ppm}$ and fragment mass tolerance set to $0.02 \mathrm{Da}$. Demamidation (NQ), oxidation (M) allowed as dynamic modifications. Peptide validation was performed using the Target-Decoy PSM validator node. The search results were imported into Scaffold Q+ for further validation (www.proteomesoftware.com) and statistical testing. A t-test was performed on the datasets and the total spectra quantitative method used to compare the datasets.

\section{Statistics}

Statistical analysis was done using Graphpad Prism 8 (version 8.4.3). All data were subjected to Shapiro-Wilks normality tests. An unpaired T-test was performed on parametric data with the data expressed as mean \pm standard deviation, whereas the Mann-Whitney $U$ test was used on unpaired non-parametric data and the data expressed as median [Q1-Q3] (all two-tailed).

\section{Supplementary material and raw data}

All supplementary material and raw data can be accessed here: https://1drv.ms/u/s!AgoCOmY3bkKHi4M_1rPgX qXoq1XXSw?e=Uq0at7.

\section{Results}

\section{Viscosity analysis of platelet poor plasma (expressed} as $\mathrm{mPa}$ /seconds)

In the current analysis, we analysed PPP from 13 controls, 10 T2DM, 13 acute COVID-19 and 11 Long COVID/ PASC patients. Data was normally distributed and unpaired T-tests showed that there were no differences in PPP viscosity of controls and T2DM $(\mathrm{p}=0.3)$, and that of controls and Long COVID/PASC PPP samples $(\mathrm{p}=0.9)$. A significant difference was noted between PPP viscosity of controls and acute COVID-19 $(\mathrm{p}=0.001)$ and acute COVID-19 and Long COVID/PASC $(\mathrm{p}=0.002)$. These results suggest that the viscosity of PPP from patients with acute COVID-19 were the only samples showed an increase plasma viscosity. Raw data files and graphs are available in the data link (see raw data link).

\section{Serum Amyloid A ELISA analysis of platelet poor plasma}

In the current analysis, we analysed PPP from 12 controls, 11 acute COVID-19 and 11 Long COVID/PASC patients. We were specifically interested in SAA1 concentrations in plasma. The human SAA protein family comprises the acute phase SAA1/SAA2, known to activate a large set of innate and adaptive immune cells, and the constitutive SAA4 [39]. ELISA analysis of SAA (1 to 4) is usually performed using serum samples. Serum SAA concentrations have recently been shown to be significantly and positively associated with higher COVID-19 severity and mortality [45]. For a comprehensive review on SAA, see [46]. Reports of serum SAA concentrations in COVID-19 
patients are between 10 and $300 \mathrm{mg} \mathrm{L}^{-1}$ (SAA type not indicated) [47]; and can be over $200 \mathrm{mg} \mathrm{L}^{-1}$ for SAA1 [48]. We analysed our data with the Mann-Whitney test (non-parametric data) or unpaired T-test (parametric data). The concentration distribution of SAA1 were as follows: for controls $\left(3.0 \mathrm{mg} \mathrm{L}^{-1}( \pm 0.9)\right)$, acute COVID19 (3.7 $\mathrm{mg} \mathrm{L}^{-1}$ [3.3-5.4]) and Long COVID/PASC (3.98 $\mathrm{mg} \mathrm{L}^{-1}( \pm 0.43)$. There was a significant difference in PPP SAA1 concentrations between controls and acute COVID-19 $(\mathrm{p}=0.02)$, and controls and Long COVID/ PASC $(p=0.003)$. There were no significant differences between PPP SAA1 concentrations acute COVID-19 and Long COVID/PASC $(\mathrm{p}=0.46)$. Raw data files and graphs are available in the data link (see raw data link below).

Platelet pathology as viewed with fluorescence microscopy Previously, we (and others) noted that platelets are hyperactivated in T2DM. Recently, we also confirmed this observation in COVID-19 samples [9]. Here we show that, in the current sample, platelets from Long COVID/PASC patients are also hyperactivated (Fig. 2E, $\mathrm{F})$, with an addition feature of clumped together platelets (Fig. 2G, H).

Platelet poor plasma (PPP): Amyloid fibrin(ogen) protein and anomalous clotting in platelet poor plasma samples, before and after two trypsin digestion protocols

Previously we have shown that naive PPP (exposed to ThT) from healthy individuals and T2DM, have significantly less anomalous microclots, compared to acute COVID-19 PPP [8]. The marker thioflavin T (ThT) binds to anomalous (amyloid) microclots in the PPP [8]. Here we show that PPP from Long COVID/PASC samples also have considerable anomalous (amyloid) microclots, similar to that of acute COVID-19 PPP samples. Figure 3 shows the naïve sample of a volunteer before COVID-19 infection and during Long COVID/PASC (Fig. 3A, B) and Fig. 3C shows more examples of microclots in other Long COVID/PASC patient PPP samples.

Plasma samples of healthy individuals, T2DM, COVID19 and Long COVID/PASC patients were then exposed to the first trypsin protocol. From each sample the supernatant was removed and ThT was added to $10 \mu \mathrm{L}$ of the remaining sample and viewed with fluorescence microscopy are shown in Figs. 4 and 5. In this remaining $10 \mu \mathrm{L}$, COVID-19 and Long COVID/PASC PPP samples, a visible pellet was present.

\section{Proteomics of platelet poor plasma after two trypsin digestion steps}

In this paper we focus only on selected proteomics results of interest for clotting; e.g. fibrinogen, von Willebrand Factor (VWF), SAA4, and plasminogen

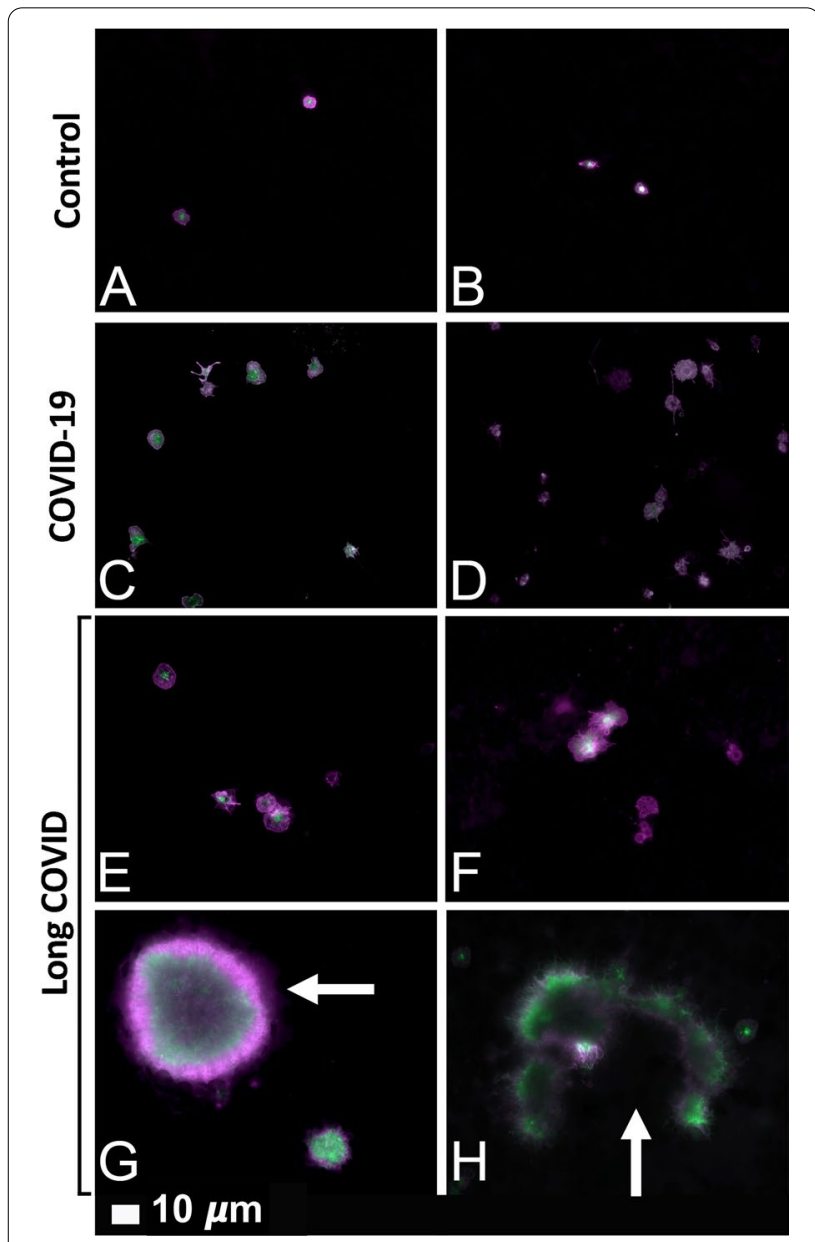

Fig. 2 Fluorescence microscopy of haematocrit samples, showing representative micrographs of platelets. A, B show representative micrographs from healthy individuals. $\mathbf{C}, \mathbf{D}$ show hyperactivated platelets in COVID-19 and $\mathbf{E}-\mathbf{H}$ show representative micrographs from platelets from Long COVID/PASC samples. White arrows show aggregated platelets. Platelets in the haematocrit were incubated with the fluorescent markers PAC-1 (green fluorescence) and CD62P-PE (purple fluorescence)

and $\alpha 2 A P$. After the first trypsinization, the PPP of the controls were fully digested, while a fibrinolyticresistant deposit was left in the COVID-19 and Long COVID/PASC samples. Only after a second trypsinization, the pellet deposit of the COVID-19 and Long COVID/PASC could be fully digested. Proteomics were performed and our results show that various inflammatory molecules that were substantially increased in the supernatant (after first trypsinization) of COVID19 and Long COVID/PASC samples compared to the supernatant from the controls. After the second trypsinization step, various inflammatory molecules were also substantially increased in the digested pellet deposits from the COVID-19 and Long COVID/ 


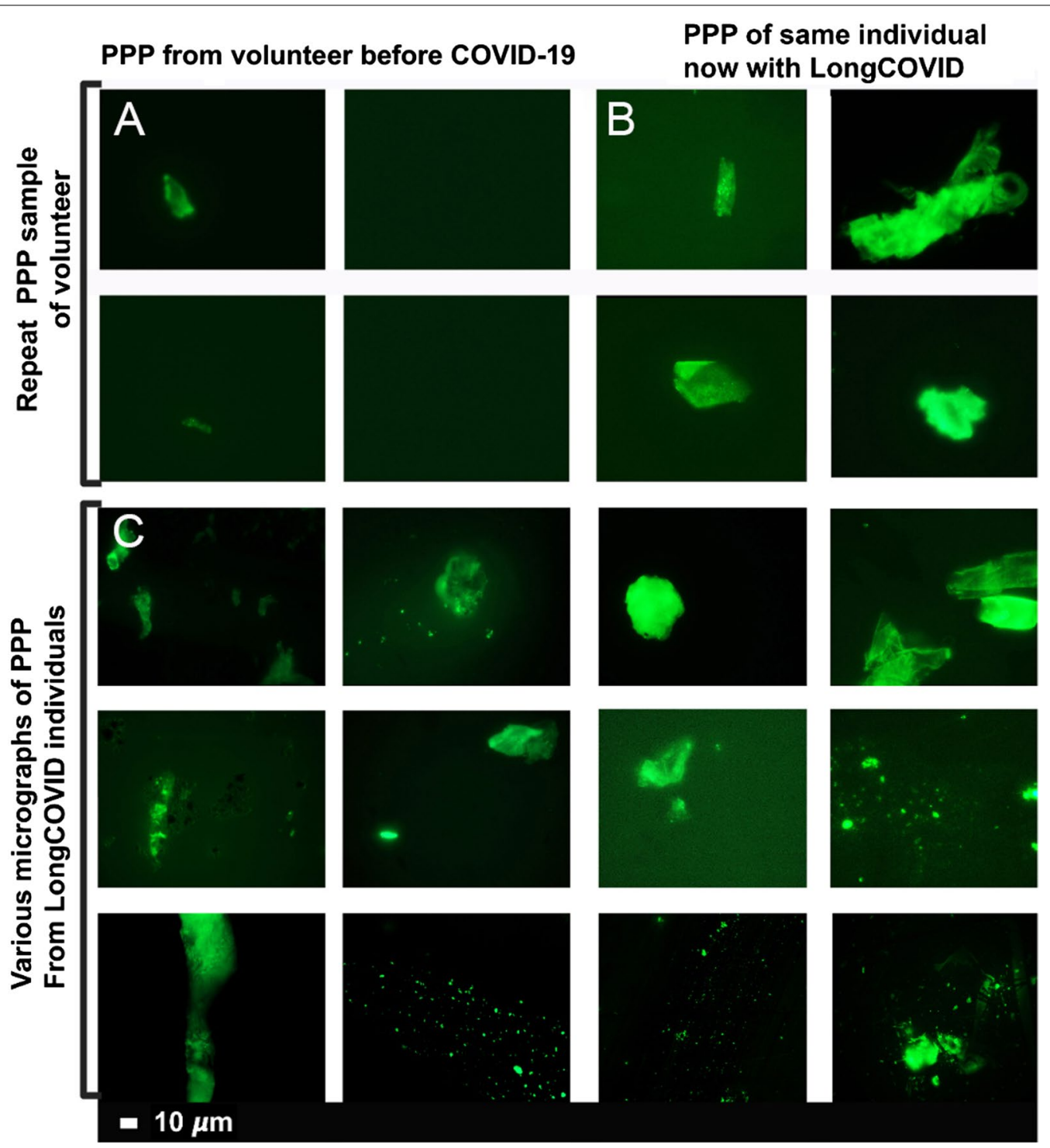

Fig. 3 Micrographs of platelet poor plasma (PPP) (BEFORE trypsin digestion) with added thioflavin T (ThT). The marker thioflavin T (ThT) binds to anomalous (amyloid) microclots in the PPP. A Microclots in a healthy volunteer, before acute COVID-19 infection and $\mathbf{B}$ the same individual during Long COVID/PASC. C Representative micrographs of other patients with Long COVID/PASC

PASC compared to the control samples. We present our results as fold changes in levels of proteins. See Table 1 for some of the most interesting results of the digested pellet deposits, as shown by fold changes (of more than 2) for the most significant proteins for pairwise comparisons. See Fig. 6 for overview plots of the protein distribution between pairwise sample comparisons (controls vs COVID-19; Controls vs Long COVID/ PASC; COVID-19 vs Long COVID/PASC). All raw data for the supernatant and digested pellet data are shown in supplementary material.

\section{Mass spectrometry of the digested platelet poor plasma} supernatants

Mass spectrometry based proteomics confirmed that the concentration of the $\alpha$-fibrinogen chain is increased in the COVID-19 supernatant compared to controls and Long COVID/PASC. The $\gamma$ and $\beta$ chains levels were not changed in the supernatant of any of the sample types. Antiplasmin and plasminogen levels were similar in the supernatant of the controls, COVID-19 and Long COVID/PASC. 


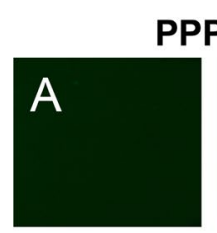

PPP from Controls
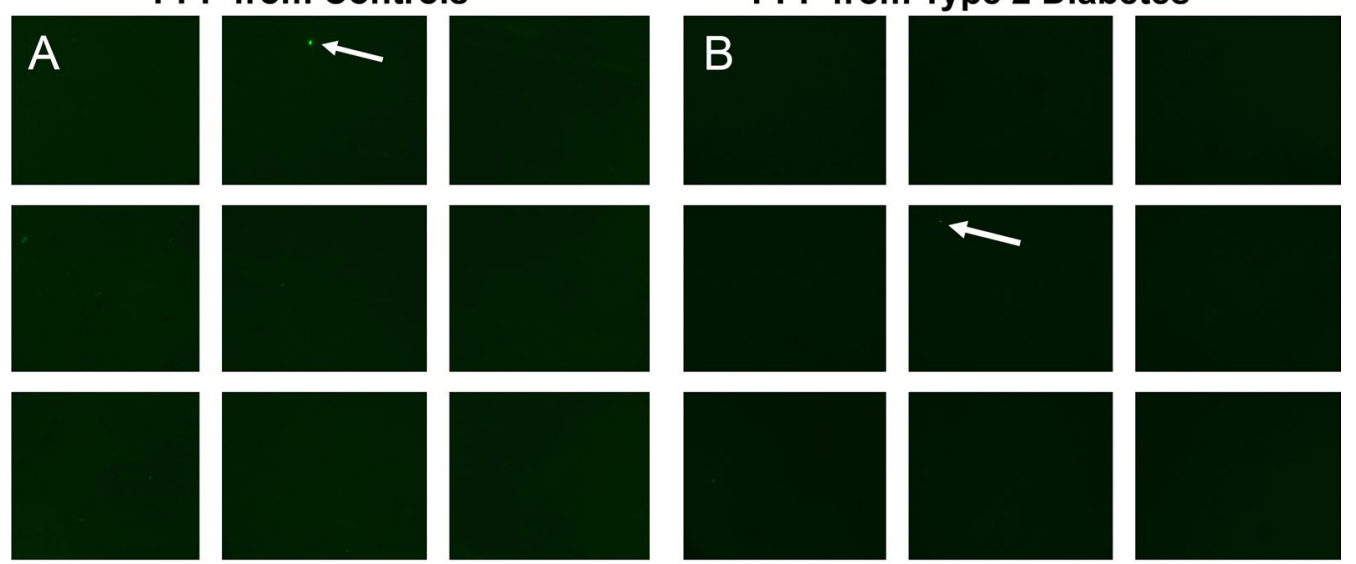

PPP from acute COVID-19
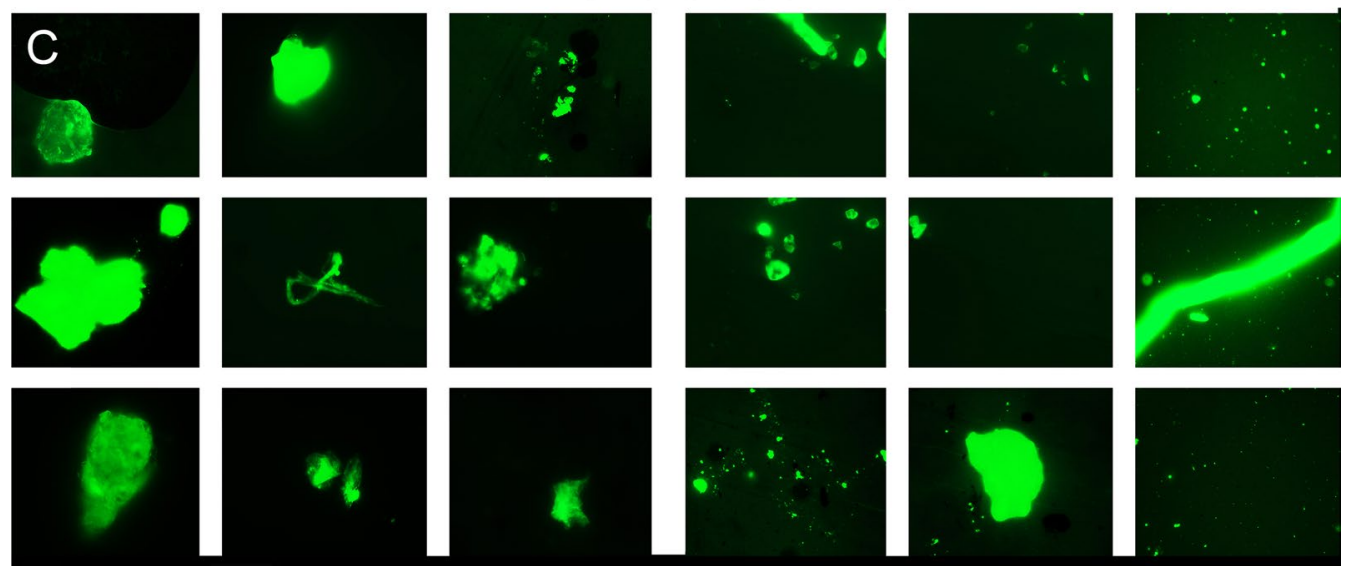

. $10 \mu \mathrm{m}$

Fig. 4 Digested supernatant of platelet poor plasma (PPP) (AFTER trypsin digestion). The marker thioflavin T (ThT) binds to anomalous (amyloid) microclots in the PPP. A Micrographs of PPP from healthy individuals; B Micrographs of PPP from Type 2 Diabetes Mellitus (T2DM). White arrows point out small areas where ThT bound in samples from controls and T2DM. C Significant microclots are visible in plasma from patients with COVID-19 as seen in the green signal in the micrographs of PPP from COVID-19

\section{Mass spectrometry of the digested pellet deposits vs} the digested faction found in control platelet poor plasma

Mass spectrometry based proteomics confirmed that levels of the coagulation factor XIII, $\alpha$ and $\beta$ fibrinogen chains were increased in the digested pellet deposits from Long COVID/PASC samples compared to the digested plasma from controls and the digested pellet deposits from COVID-19. Relative to control and COVID-19 samples, plasminogen levels were slightly increased in the pellet deposits from Long COVID/ PASC. SAA4 was not present in the digested plasma of the controls, but present in the digested pellet deposits of samples from Long COVID/PASC. It was a surprising result that SAA4 was so much increased in the digested pellet deposits. We suggest that these molecules are trapped and concentrated in the fibrinolysisresistant microclots that are present in the circulation. SAA4 is a constitutively expressed molecule in contrast to SAA1 and 2 that are both acute phase proteins.

\section{Mass spectrometry of the digested platelet poor plasma} supernatants controls vs Type 2 Diabetes and Type 2

\section{Diabetes vs Long COVID samples}

PPP of T2DM and controls digested fully after the 1st trypsinization step. Proteomics of supernatants showed fold-change increases for Complement $\mathrm{C} 1 \mathrm{r}$ subcomponent-like protein and SAA1 in the T2DM digested sample than in the control sample. SAA1 is known to be increased in T2DM [49]. The classical complement 

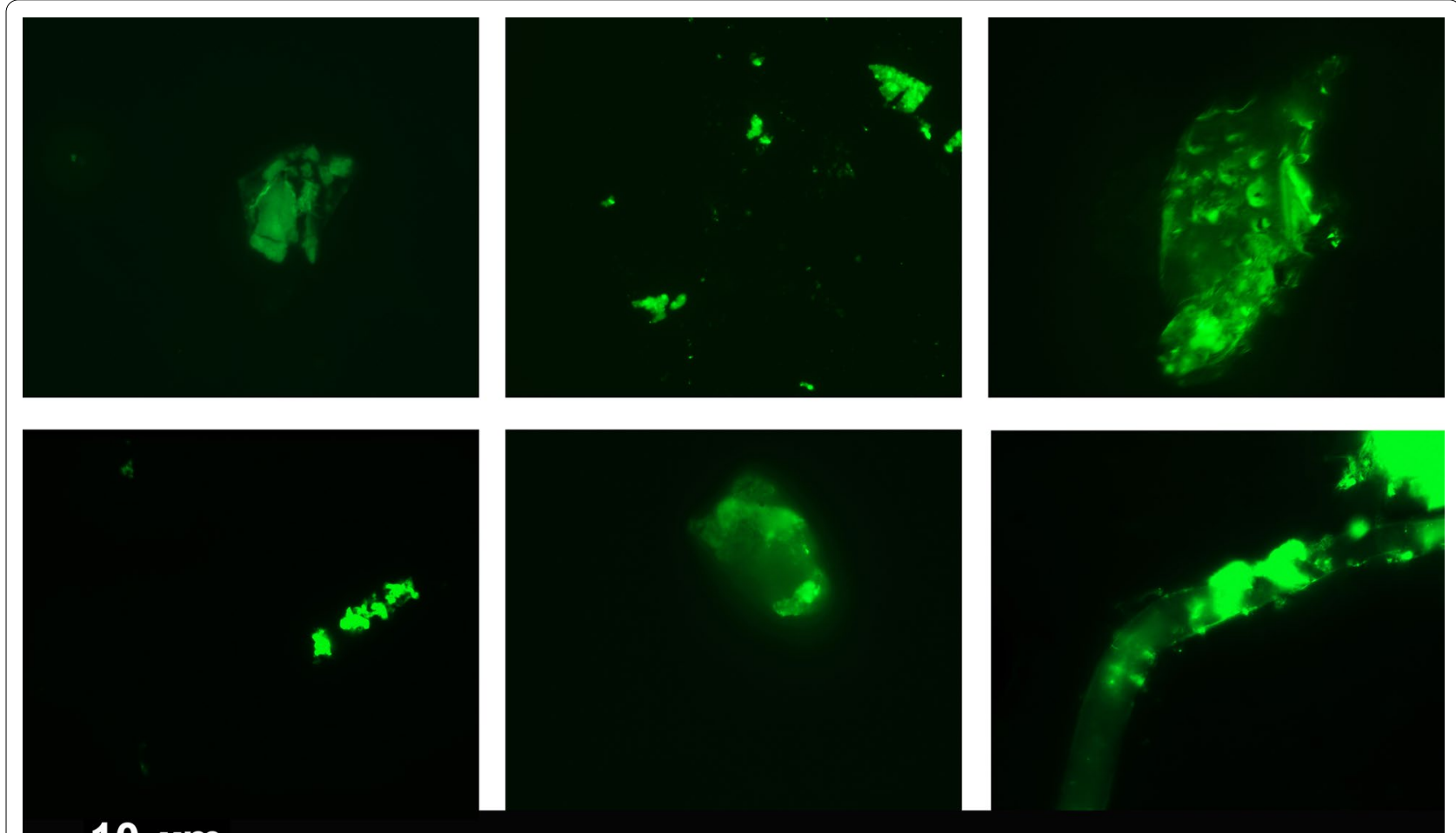

Fig. 5 Representative micrographs of Long COVID/PASC patients. Digested supernatant (PPP) after first trypsin digestion step, where supernatant was removed and thioflavin T (ThT) added to the remaining $10 \mu \mathrm{L}$. The marker thioflavin T (ThT) binds to anomalous microclots in the PPP

pathway activation is a multimolecular complex, where three subcomponents $\mathrm{C} 1 \mathrm{q}, \mathrm{C} 1 \mathrm{r}$, and $\mathrm{C} 1 \mathrm{~s}$. $\mathrm{C} 1 \mathrm{q}$ are present. [50]. These subunits form the recognition areas of the complex and its binding to specific targets leads to the formation of active $\mathrm{C}$, which in turn activates the classical complement pathway in an immunoglobulindependent or -independent manner [50].

We also compared the supernatant after the 1st trypsinization step of T2DM and Long COVID. Here we found significant fold changes in $\mathrm{C} 1 \mathrm{q}$ subcomponent subunit $\mathrm{B}$, and two types of Keratin, type I and II (cytoskeletal).

\section{Discussion}

It is now well-recognized that vascular changes and thrombotic microangiopathy, diffuse intravascular coagulation and large-vessel thrombosis are major reasons for a poor COVID-19 prognosis [51, 52]. These comorbidities are linked to multisystem organ failure, as well as pulmonary vascular endothelialitis [51, 53]. The presence of endotheliopathy in particular, is likely to be associated with critical illness and death [54]. It is also suggested that endothelial dysfunction contributes to COVID-19-associated vascular inflammation,
COVID-19-associated coagulopathy, and pulmonary fibrinous microthrombi in the alveolar capillaries [53]. In some instances, patients present with a significant elevation in D-dimer/fibrin(ogen)degradation products [55]. D-dimer and fibrin(ogen) degradation products may indicate the failing attempt of the fibrinolytic system to remove fibrin and necrotic tissue from the lung parenchyma (and also from the circulation), but being consumed or overwhelmed in the process [56].

Central to COVID-19 pathology is the pathological roller-coaster from hypercoagulation and hypofibrinolysis. Bouck and co-workers in 2021 found that the lag times to thrombin, plasmin, and fibrin formation were prolonged with increased disease severity in COVID-19 [57]. The authors also argue that, although the presence of D-dimer suggests fibrinolytic pathways are intact and actively dissolving (lysing) fibrin, the discovery of fibrin deposits in lungs and other organs suggests dysregulation of the balance in fibrin-forming and fibrin-dissolving (plasmin generation) pathways is a major aspect of COVID-19 pathogenesis [57].

Results presented in the current paper point to a significant failure in the fibrinolytic process during COVID19 and also in patients with lingering Long COVID/ 


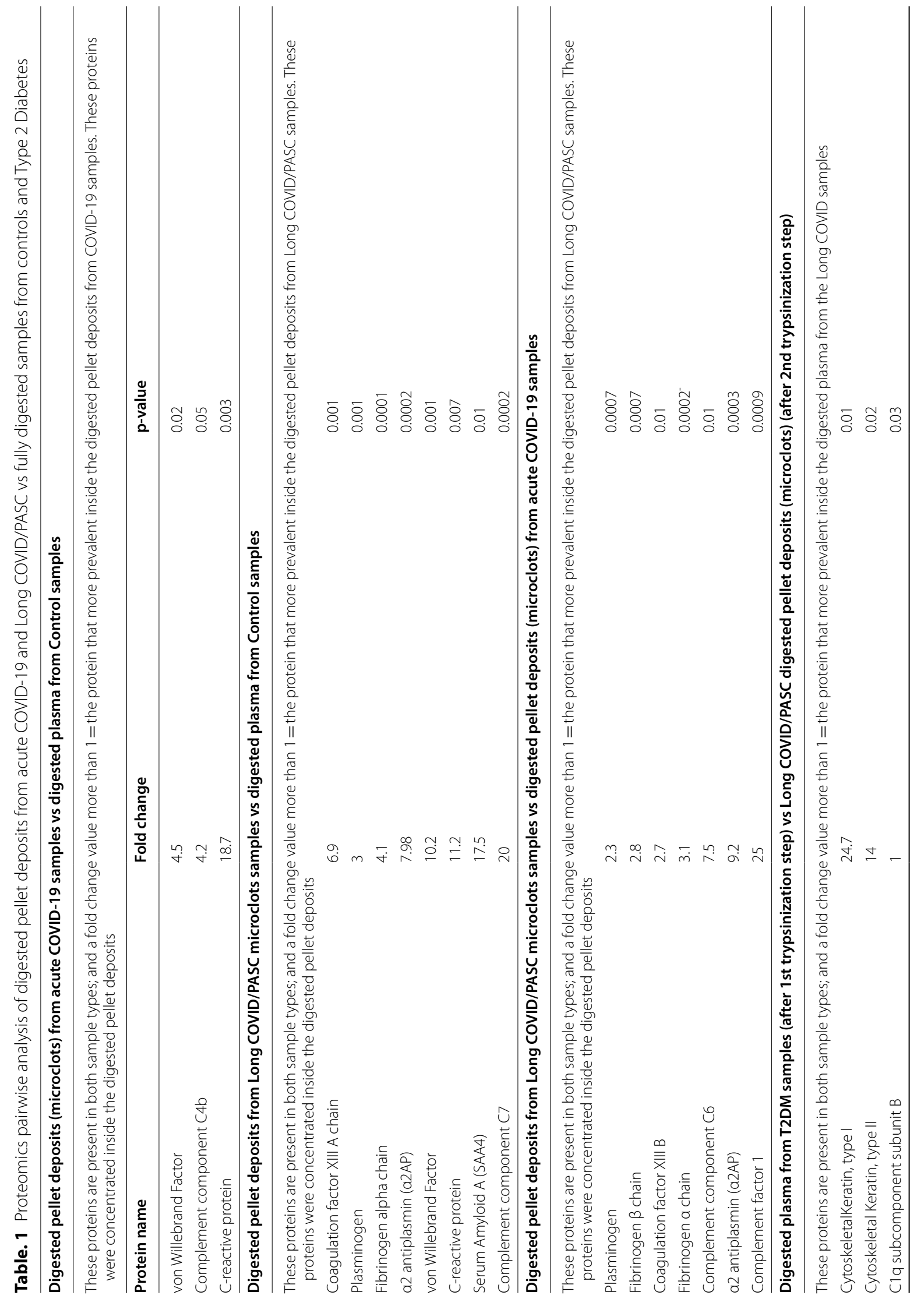




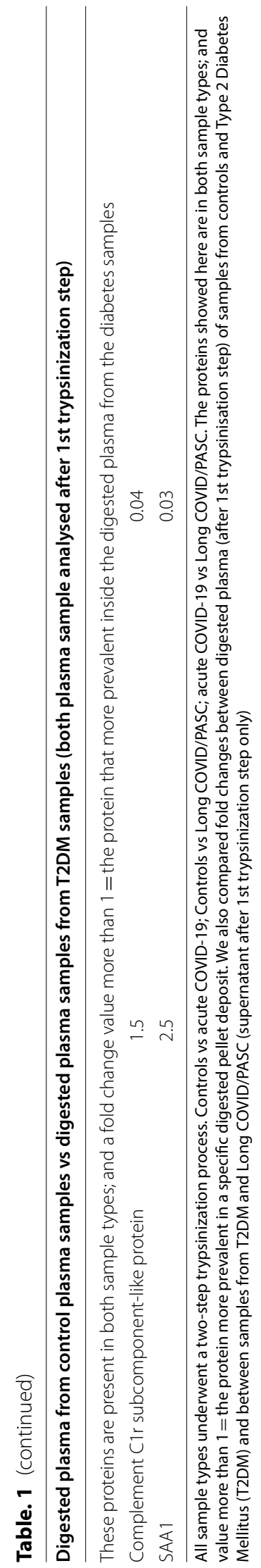




\section{A: Acute COVID-19 vs Control}

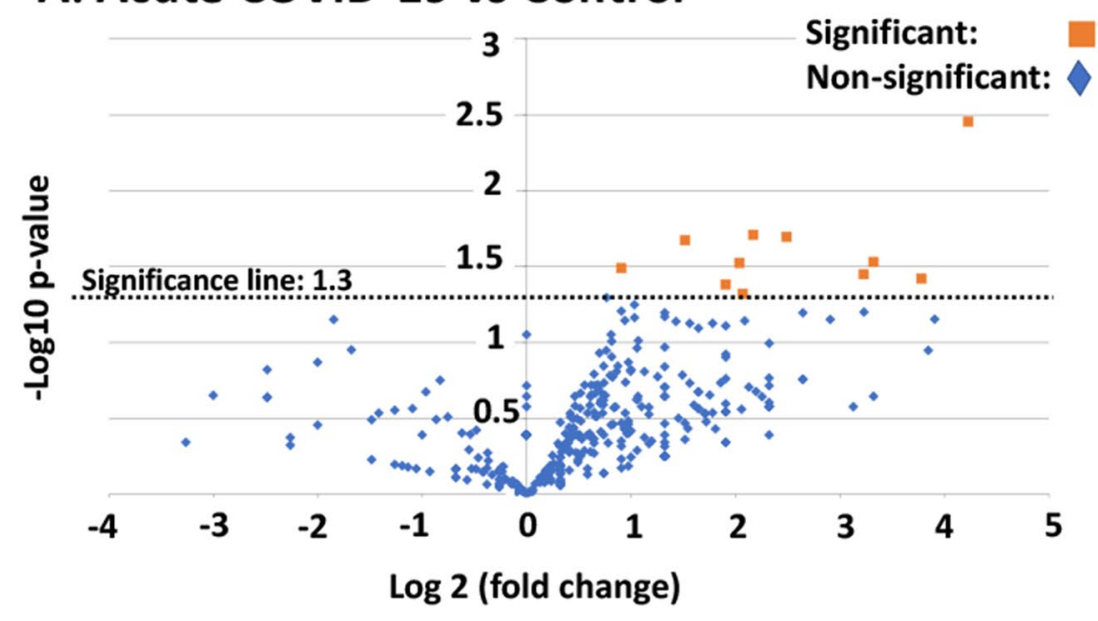

\section{B: Long COVID vs Control}

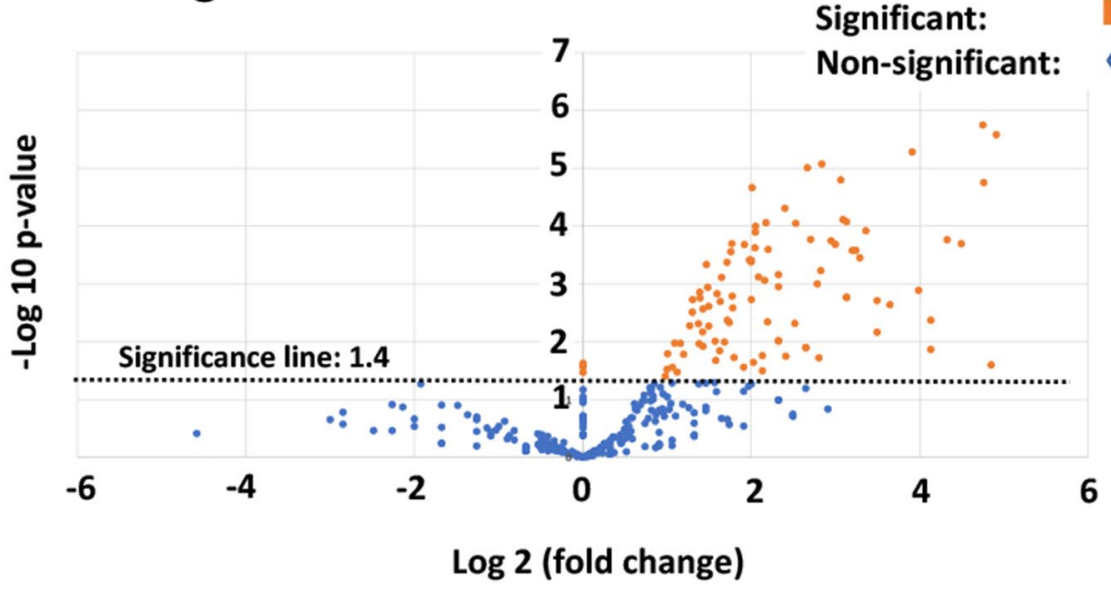

C: Long COVID vs Acute Covid-19

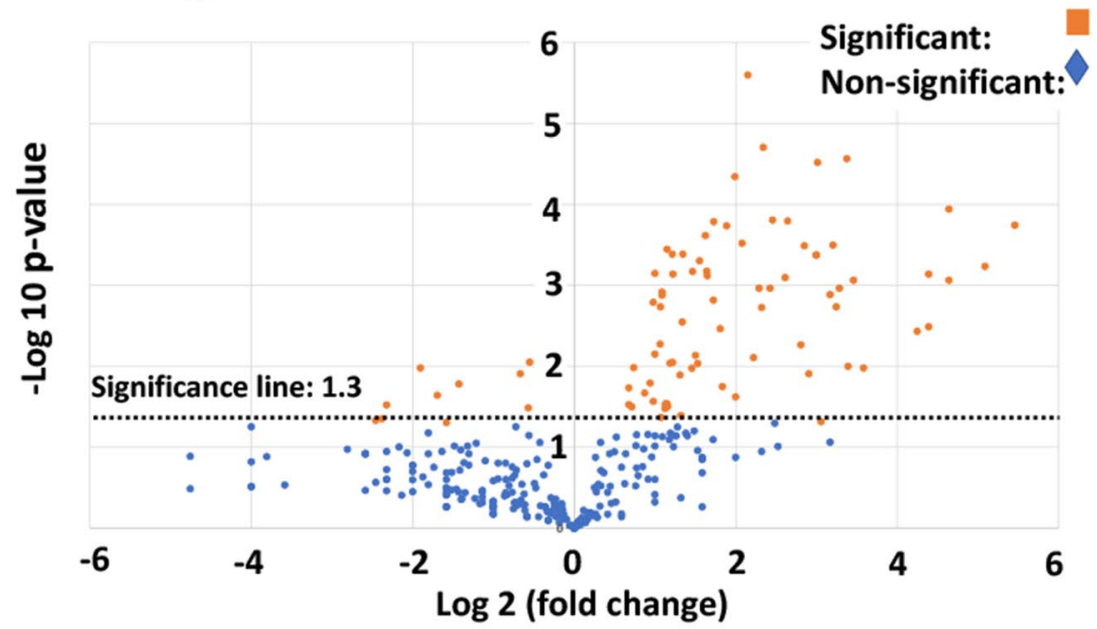

Fig. 6 Volcano plots of the protein distribution between pairwise sample comparisons (controls vs COVID-19; controls vs Long COVID/PASC; COVID-19 vs Long COVID/PASC). Oranges dots show proteins above the significance levels as indicated by the dotted line. Foldchange is presented on the $X$-axis (negative values: down-regulation and positive values: upregulation). The Y-axis represents the Log-(minus) 10 of the $p$-values 
PASC symptoms. Our results show that plasma proteins in both COVID-19 and Long COVID/PASC plasma samples are greatly resistant to breakdown in the presence of trypsin. This was confirmed visually using fluorescence microscopy, as well as with proteomics. Most significant changes shown in proteomics analysis, were in circulating proteins related to clotting. We noted significant increases in fibrinogen chains, as well as acute phase proteins like SAA4 and $\alpha 2 \mathrm{AP}$, as shown in the proteomics analysis (Table 1). Our results point to a significant increase in $\alpha 2 \mathrm{AP}$ in Long COVID/PASC vs controls and in Long COVID/PASC vs acute COVID-19 for digested pellet deposits. Here we also show a difference between SAA1 and SAA4. SAA1, was about twofold increased in PPP from both acute COVID-19 and Long COVID/PASC as seen with an SAA1 ELISA. SAA1 and Complement $\mathrm{C} 1 \mathrm{r}$ subcomponent-like protein were also increased in the fully digested plasma supernatant of T2DM (after a first trypsinization step), compared to the fully digested plasma supernatant samples from controls. Increases in SAA1 is known in T2DM [49], and adverse vascular effects of complement, the inflammatory process, and a thrombotic environment may play significant roles in T2DM pathology [58]. Future analysis could also investigate the role of other complement molecules e.g. C3, C6 and C7 in acute and acute Long COVID/PASC. We also found increases in VWF in COVID-19 and Long COVID/PASC samples. This is of significance for platelet adhesion to endothelium [59]. When VWF is higher, platelets will be more activated and more prone to adhesion to endothelium [60].

SAA4 (also found as an apoliprotein of HDL), is synthesized constitutively in the liver [46]. Here we report that SAA4 showed a significant increase in our proteomics analysis of the double trypsin-digested pellet deposits. There was a 17 -fold increase in SAA4, between PPP in samples from Long COVID/PASC vs controls. We report fold changes in proteins present in the fibrinolysis-resistant pellet deposits of acute COVID-19 and Long COVID/ PASC, compared to the fluid sample of the controls that also underwent a double trypsinization process. It follows that the true concentrations in plasma samples may therefore not reflect the fold changes we report on here, in proteins trapped in the solubilized pellet deposits. It was recently shown that patients with low HDL-cholesterol levels at admission to the hospital were more likely to develop severe disease, compared to patients with high HDL-cholesterol levels [61]. Compared with the healthy controls, the patients have sharply decreased concentrations of total cholesterol, HDL-cholesterol and LDLcholesterol [62]. With reduced HDL levels in circulation, SAA4 potentially will be less partitioned into HDL.
Of particular interest is the simultaneous presence of persistent anomalous (amyloid) microclots and a pathological fibrinolytic system. The plasmin and antiplasmin balance may be central to this phenomenon (see Fig. 7). An important element of the fibrinolytic system is the conversion of circulating zymogen plasminogen to its active form plasmin [63, 64]. Endogenous activators of plasminogen are the tissue-type plasminogen activator (tPA) and urokinase-type plasminogen activator (uPA) [65]. The catalytic activity of tPA is largely dependent on the presence of fibrin, as both IPA and its substrate plasminogen bind to the lysine residues on fibrin, using it as a cofactor for plasmin generation [64]. Plasmin is the effector protease of the fibrinolytic system, well known for its involvement in fibrin degradation and clot removal [63]. Plasmin is also recognized as a potent modulator of immunological processes by directly interacting with various cell types including cells of the vasculature (endothelial cells, smooth muscle cells) [63] In fact, the removal of misfolded proteins and the maintenance of tissue homeostasis seem to be major physiological functions of plasmin [63]. Plasmin is also inhibited by the actions of various serine protease inhibitors like $\alpha 2 \mathrm{AP}$ [64]. High blood levels of $\alpha 2 \mathrm{AP}$ [66], an ultrafast, covalent inhibitor of plasmin, have been linked in humans to increased risk of ischemic stroke and failure of tissue plasminogen activator therapy [67]. Furthermore, plasminogen activator inhibitor-1 (PAI-1) and $\alpha 2 \mathrm{AP}$, maintain a delicate homeostasis in the normal physiologic state [68]. $\alpha 2 \mathrm{AP}$ is covalently cross-linked to fibrin in the thrombus by activated factor XIII, a transglutaminase $[69,70]$ which is a major source of the resistance of in vitro plasma clots to plasmin-mediated fibrinolysis [66]. It is therefore entirely plausible that, as we noted in our Long COVID/ PASC samples, if there is both an acute or lingering overload of anomalous (amyloid) fibrin(ogen) microclots in circulation, and a significant fold increase in $\alpha 2 \mathrm{AP}$, that the endogenous activators of plasminogen and the subsequent cascade of physiological events that are driven by plasmin generation, will fail. In addition, plasmin also processes the viral S-protein for its entry into the host cells [65], where plasmin, and other proteases cleave a newly inserted furin site in the S protein of SARS-CoV-2, extracellularly, which increases its infectivity and virulence [56]. This involvement of plasmin in the cleaving of the viral $\mathrm{S}$ proteins, may also further contribute to a decreased efficiency of plasmin to act on an increase in anomalous fibrin(ogen) load.

\section{Conclusion}

Hypercoagulability is a well-known accompaniment of T2DM [49, 73]. Furthermore, hypercoagulability is an increasingly recognized complication of COVID-19 


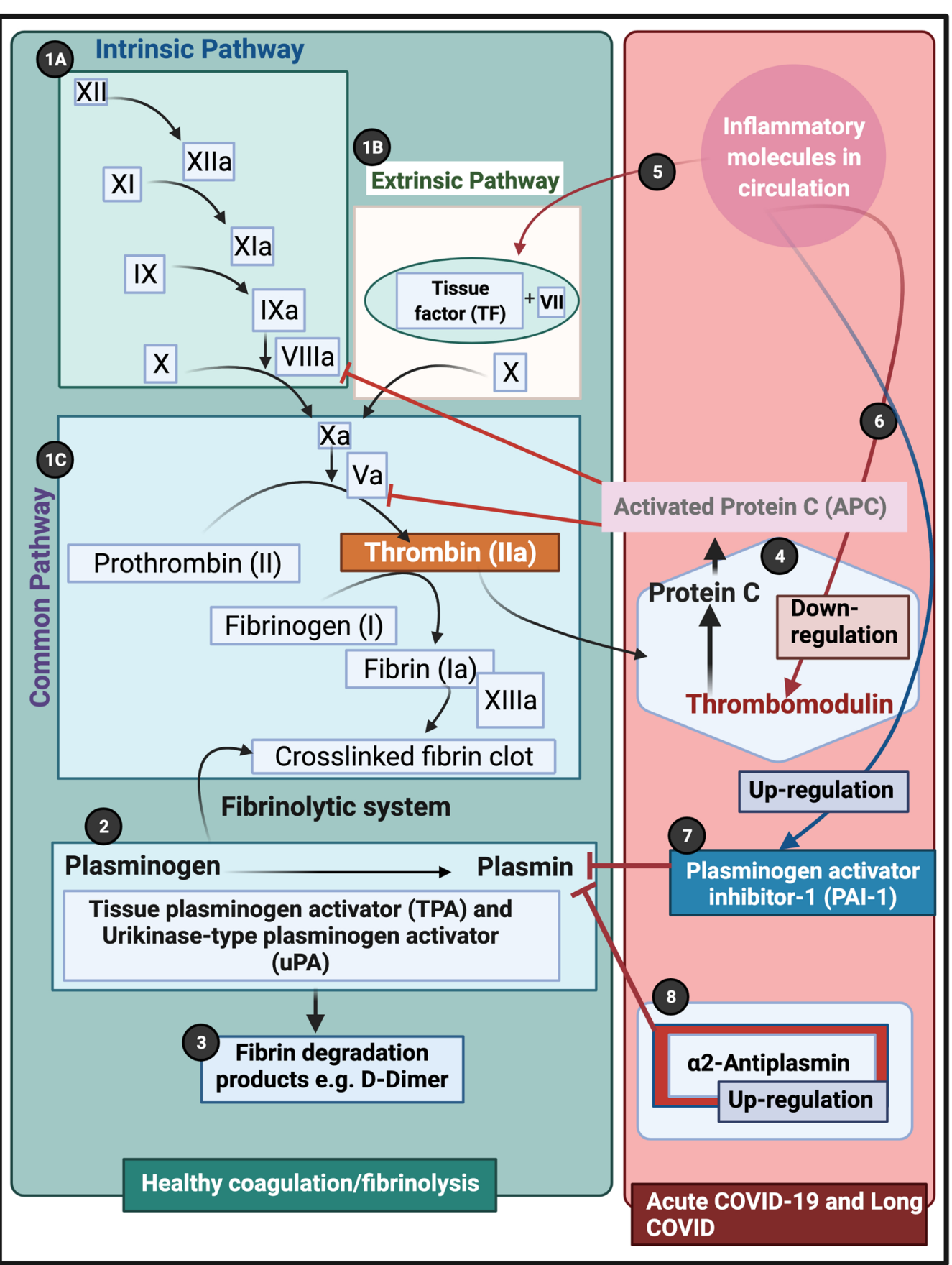

Fig. 7 Simplified coagulation diagram (adapted from [37, 71, 72] depicting healthy and pathological processes. (1A) The intrinsic and (1B) extrinsic pathways converge into the (1C) common pathway. These pathways lead to the conversion of soluble fibrinogen to insoluble fibrin, catalysed by thrombin. (2) Tissue plasminogen activator (tPA) or urokinase-type plasminogen activator (UPA) converts plasminogen into plasmin. A healthy fibrinolytic system regulates the coagulation pathway and assists with successful lysis of the insoluble fibrin clot. (3) Plasmin cleaves fibrin into fibrin degradation products (FDPs), including D-dimer. (4) Protein C and thrombomodulin both regulate coagulation: thrombin binds to its receptor, thrombomodulin, resulting in activated protein C (APC). APC then inhibits both Va and VIIIa. (5) Dysregulated inflammatory molecules may interfere with tissue factor (TF) expression. (6) Dysregulated inflammatory molecules may also down-regulate thrombomodulin, resulting in hypercoagulation, as Va and VIIla activities are then not sufficiently modulated. (7) Dysregulated inflammatory molecules in circulation can inhibit of the fibrinolytic system via up-regulation of plasminogen activator inhibitor-1 (PAI-1). PAl-I upregulation interferes with tissue plasminogen activator (TPA) function, and ultimately results in a dysregulated coagulation system. (8) a2-antiplasmin (a2AP) inhibits plasmin and ultimately will prevent sufficient fibrinolysis to happen. (Figure created with Biorender.com) 
infection, and anticoagulation has become central in the comprehensive COVID-19 management [65, 74]. Significant anomalous (amyloid) microclot formation that are resistant to fibrinolysis, increased $\alpha 2 \mathrm{AP}$ and the surge of acute phase inflammatory molecules, may therefore be central contributors in the multiple coagulation/fibrinolysis pathophysiology of both COVID-19 infection and its lingering phenotype, Long COVID/PASC. Our findings that there are significant increases of dysregulated molecules when comparing acute COVID-19 samples to Long COVID/PASC, was interesting, and we speculate that it might be because of the prolonged inflammatory status in Long COVID/PASC, and could also possibly be due to persistent viral infection (although this was not confirmed in the current study). We recognize that some of the proteomics findings should now be confirmed using a larger sample set as the statistical power of the current set is limited. We also suggest that methods like platelet aggregometry analysis and PT and PTT analysis should also be considered. Here we conclude that (i) hypercoagulability due to significant increases in inflammatory molecules, (ii) circulating microclots and hyperactivated platelets, and (iii) an aberrant fibrinolytic system, are all driven by a dysfunction in clotting protein and lytic enzyme supply and demand. Central to hypofibrinolysis and persistent microclots is the presence of a significant increase in $\alpha 2 \mathrm{AP}$. (iv) The association of T2DM and the development of Long COVID/PASC needs urgent attention and further research is needed to determine the association between T2DM and Long COVID/PASC. Clotting pathologies in both acute COVID-19 infection and in Long COVID/PASC might therefore benefit from a following a regime of continued anticlotting therapy to support the fibrinolytic system function.

\section{Acknowledgements}

We would like to thank the patients and their families who participated in this study. We wish to thank Simone Turner and Mireille Grobler for their assistance with the ELISA assay and assistance with the curation of the samples. We also wish to thank Dr Amy Proal from PolyBio Research Foundation for comments on the paper.

\section{Authors' contributions}

EP: Sample analysis, edited the paper, funding, co-corresponding author; MV: proteomics analysis and proteomics data curation; CV: Platelet analysis and technical assistance; JAB: ELISA analysis; PJL: Clinician and patient sample identification; JS: Haematopathologist: sample collection and screening; DBK: cocorresponding author. . All authors read and approved the final manuscript.

\section{Funding}

DBK thanks the Novo Nordisk Foundation for financial support (grant NNF20CC0035580). The funders had no role in study design, data collection and analysis, decision to publish, or preparation of the manuscript.

\section{Availability of data and materials}

The datasets generated as well as figure micrographs analyzed during the current study are available: https://1drv.ms/u/s!AgoCOmY3bkKHi4M_1rPgX qXoq1XXSw? $=$ Uq0at7. The raw data supporting the conclusions of this article will be made available by the authors, without undue reservation.

\section{Declarations}

\section{Ethics approval and consent to participate}

Ethical approval for blood collection and analysis of the patients with COVID19, Long COVID/PASC and T2DM and healthy individuals, was given by the Health Research Ethics Committee (HREC) of Stellenbosch University (reference number: 9521). This laboratory study was carried out in strict adherence to the International Declaration of Helsinki, South African Guidelines for Good Clinical Practice and the South African Medical Research Council (SAMRC), Ethical Guidelines for research. Consent was obtained from all participant. Patients or the public WERE NOT involved in the design, or conduct, or reporting, or dissemination plans of our research.

\section{Consent for publication}

All authors approved submission of the paper.

\section{Competing interests}

The authors have no competing interests to declare.

\section{Author details}

${ }^{1}$ Department of Physiological Sciences, Faculty of Science, Stellenbosch University, Private Bag X1 Matieland, Stellenbosch 7602, South Africa. ${ }^{2}$ Central Analytical Facility: Mass Spectrometry, Stellenbosch University, Tygerberg Campus, Room 6054, Clinical Building, Francie Van Zijl Drive Tygerberg, Cape Town 7505, South Africa. ${ }^{3}$ Mediclinic Stellenbosch, Stellenbosch 7600, South Africa. ${ }^{4}$ PathCare Laboratories, PathCare Business Centre, PathCare Park, Neels Bothma Street, N1 City, Cape Town 7460, South Africa. ${ }^{5}$ Department of Biochemistry and Systems Biology, Institute of Systems, Molecular and Integrative Biology, Faculty of Health and Life Sciences, University of Liverpool, Liverpool L69 7ZB, UK. ${ }^{6}$ The Novo Nordisk Foundation Centre for Biosustainability, Technical University of Denmark, Kemitorvet 200, 2800 Kgs Lyngby, Denmark.

Received: 23 June 2021 Accepted: 3 August 2021

Published online: 23 August 2021

\section{References}

1. Gupta A, Madhavan MV, Sehgal K, Nair N, Mahajan S, Sehrawat TS, Bikdeli B, Ahluwalia N, Ausiello JC, Wan EY, et al. Extrapulmonary manifestations of COVID-19. Nat Med. 2020;26(7):1017-32.

2. Perico L, Benigni A, Casiraghi F, Ng LFP, Renia L, Remuzzi G. Immunity, endothelial injury and complement-induced coagulopathy in COVID-19. Nat Rev Nephrol. 2021;17(1):46-64.

3. Gerotziafas GT, Catalano M, Colgan MP, Pecsvarady Z, Wautrecht JC, Fazeli B, Olinic DM, Farkas K, Elalamy I, Falanga A, et al. Guidance for the management of patients with vascular disease or cardiovascular risk factors and COVID-19: position paper from VAS-European independent foundation in angiology/vascular medicine. Thromb Haemost. 2020;120(12):1597-628.

4. Siddiqi HK, Libby P, Ridker PM. COVID-19—a vascular disease. Trends Cardiovasc Med. 2021:31(1):1-5.

5. Grobbelaar LM, Venter C, Vlok M, Ngoepe M, Laubscher GJ, Lourens PJ, Steenkamp J, Kell DB, Pretorius E. SARS-CoV-2 spike protein S1 induces fibrin(ogen) resistant to fibrinolysis: Implications for microclot formation in COVID-19. MedRxiv. 2021. https://doi.org/10.1101/2021.03.05.21252 960.

6. Grobler C, Maphumulo SC, Grobbelaar LM, Bredenkamp JC, Laubscher GJ Lourens PJ, Steenkamp J, Kell DB, Pretorius E. Covid-19: the rollercoaster of fibrin(Ogen), D-dimer, Von Willebrand factor, P-selectin and their interactions with endothelial cells, platelets and erythrocytes. Int J Mol Sci. 2020;21(14):5168.

7. Kell DB, Heyden EL, Pretorius E. The biology of lactoferrin, an iron-binding protein that can help defend against viruses and bacteria. Front Immunol. 2020;11:1221

8. Pretorius E, Venter C, Laubscher GJ, Lourens PJ, Steenkamp J, Kell DB. Prevalence of readily detected amyloid blood clots in "unclotted"Type 2 Diabetes Mellitus and COVID-19 plasma: a preliminary report. Cardiovasc Diabetol. 2020;19(1):193. 
9. Venter C, Bezuidenhout JA, Laubscher GJ, Lourens PJ, Steenkamp J, Kell DB, Pretorius E. Erythrocyte, platelet, serum ferritin, and P-selectin pathophysiology implicated in severe hypercoagulation and vascular complications in COVID-19. Int J Mol Sci. 2020;21(21):8234.

10. Roberts I, Muelas MW, Taylor JM, Davison AS, Xu Y, Grixti JM, Gotts N, Sorokin A, Goodacre R, Kell DB. Untargeted metabolomics of COVID-19 patient serum reveals potential prognostic markers of both severity and outcome. MedRxiv. 2020. https://doi.org/10.1101/2020.12.09.20246389.

11. Renzi S, Landoni G, Zangrillo A, Ciceri F. MicroCLOTS pathophysiology in COVID 19. Korean J Intern Med. 2020. https://doi.org/10.3904/kjim.2020 336.

12. Ciceri F, Beretta L, Scandroglio AM, Colombo S, Landoni G, Ruggeri A, Peccatori J, D'Angelo A, De Cobelli F, Rovere-Querini P, et al. Microvascular COVID-19 lung vessels obstructive thromboinflammatory syndrome (MicroCLOTS): an atypical acute respiratory distress syndrome working hypothesis. Crit Care Resusc. 2020;22(2):95-7.

13. Bobrova L, Kozlovskaya N, Korotchaeva Y, Bobkova I, Kamyshova E, Moiseev S. Microvascular COVID-19 lung vessels obstructive thromboinflammatory syndrome (MicroCLOTS): a new variant of thrombotic microangiopathy? Crit Care Resusc. 2020;22(3):284

14. Lam LM, Murphy SJ, Kuri-Cervantes L, Weisman AR, Ittner CAG, Reilly JP, Pampena MB, Betts MR, Wherry EJ, Song WC, et al. Erythrocytes reveal complement activation in patients with COVID-19. MedRxiv. 2020 https://doi.org/10.1101/2020.05.20.20104398.

15. Berzuini A, Bianco C, Paccapelo C, Bertolini F, Gregato G, Cattaneo A, Erba E, Bandera A, Gori A, Lamorte G, et al. Red cell-bound antibodies and transfusion requirements in hospitalized patients with COVID-19. Blood. 2020;136(6):766-8.

16. Akhter N, Ahmad S, Alzahrani FA, Dar SA, Wahid M, Haque S, Bhatia K Sr, Almalki S, Alharbi RA, Sindi AAA. Impact of COVID-19 on the cerebrovascular system and the prevention of RBC lysis. Eur Rev Med Pharmacol Sci. 2020;24(19):10267-78.

17. Terpos E, Ntanasis-Stathopoulos I, Elalamy I, Kastritis E, Sergentanis TN, Politou M, Psaltopoulou T, Gerotziafas G, Dimopoulos MA. Hematological findings and complications of COVID-19. Am J Hematol. 2020;95(7):834-47.

18. Finer N, Garnett SP, Bruun JM. COVID-19 and obesity. Clin Obes. 2020;10(3):e12365.

19. Yates T, Razieh C, Zaccardi F, Davies MJ, Khunti K. Obesity and risk of COVID-19: analysis of UK biobank. Prim Care Diabetes. 2020;14(5):566-7.

20. Sattar N, Ho FK, Gill JM, Ghouri N, Gray SR, Celis-Morales CA, Katikireddi SV, Berry C, Pell JP, McMurray JJ, et al. BMI and future risk for COVID-19 infection and death across sex, age and ethnicity: Preliminary findings from UK biobank. Diabetes Metab Syndr. 2020;14(5):1149-51.

21. Li B, Yang J, Zhao F, Zhi L, Wang X, Liu L, Bi Z, Zhao Y. Prevalence and impact of cardiovascular metabolic diseases on COVID-19 in China. Clin Res Cardiol. 2020;109(5):531-8.

22. Malek AE, Raad II, Jabbour E. Cancer and COVID-19. Lancet. 2020;396(10257):1066-7.

23. Proal AD, VanElzakker MB. Long COVID or Post-acute sequelae of COVID 19 (PASC): an overview of 1 biological factors that may contribute to persistent symptoms. Front Microbiol. 2021;12:1494 (in press).

24. Lopez-Leon S, Wegman-Ostrosky T, Perelman C, Sepulveda R, Rebolledo P, Cuapio A, Villapol S. More than 50 long-term effects of COVID-19: a systematic review and meta-analysis. Res Sq. 2021. https://doi.org/10. 1101/2021.01.27.21250617.

25. Carfi A, Bernabei R, Landi F. Persistent symptoms in patients after acute COVID-19. JAMA. 2020;324(6):603-5.

26. Rubin R. As their numbers grow, COVID-19"Long Haulers" stump experts. JAMA. 2020:324:1381-3.

27. Baig AM. Chronic COVID syndrome: need for an appropriate medical terminology for long-COVID and COVID long-haulers. J Med Virol. 2020;93:2555-6.

28. Wostyn P. COVID-19 and chronic fatigue syndrome: is the worst yet to come? Med Hypotheses. 2021;146:110469.

29. Mohabbat AB, Mohabbat NML, Wight EC. Fibromyalgia and chronic fatigue syndrome in the age of COVID-19. Mayo Clin Proc Innov Qual Outcomes. 2020;4(6):764-6.

30. Goldstein DS. The possible association between COVID-19 and postural tachycardia syndrome. Heart Rhythm. 2021;18(4):508-9.
31. Afrin LB, Weinstock LB, Molderings GJ. Covid-19 hyperinflammation and post-Covid-19 illness may be rooted in mast cell activation syndrome. Int $J$ Infect Dis. 2020;100:327-32.

32. Huang C, Huang L, Wang Y, Li X, Ren L, Gu X, Kang L, Guo L, Liu M, Zhou $X$, et al. 6-month consequences of COVID-19 in patients discharged from hospital: a cohort study. Lancet. 2021;397(10270):220-32.

33. Tabacof L, Tosto-Mancuso J, Wood J, Cortes M, Kontorovich A, McCarthy D, Rizk D, Mohammadi N, Breyman E, Nasr L, et al. Post-acute COVID-19 syndrome negatively impacts health and wellbeing despite less severe acute infection. MedRxiv. 2020. https://doi.org/10.1101/2020.11.04.20226 126.

34. Mandal S, Barnett J, Brill SE, Brown JS, Denneny EK, Hare SS, Heightman M, Hillman TE, Jacob J, Jarvis HC, et al. "Long-COVID": a cross-sectional study of persisting symptoms, biomarker and imaging abnormalities following hospitalisation for COVID-19. Thorax. 2020;76:396-8.

35. Komaroff AL, Bateman L. Will COVID-19 lead to myalgic encephalomyelitis/chronic fatigue syndrome? Front Med (Lausanne). 2020;7:606824.

36. Schaller J, Gerber SS. The plasmin-antiplasmin system: structural and functional aspects. Cell Mol Life Sci. 2011;68(5):785-801.

37. Miszta A, Huskens D, Donkervoort D, Roberts MJM, Wolberg AS, de Laat B. Assessing plasmin generation in health and disease. Int J Mol Sci. 2021;22(5):2758.

38. Pretorius E, Oberholzer HM, van der Spuy WJ, Meiring JH. Smoking and coagulation: the sticky fibrin phenomenon. Ultrastruct Pathol. 2010;34(4):236-9.

39. Jumeau C, Awad F, Assrawi E, Cobret L, Duquesnoy P, Giurgea I, Valeyre D, Grateau G, Amselem S, Bernaudin JF, et al. Expression of SAA1, SAA2 and SAA4 genes in human primary monocytes and monocyte-derived macrophages. PLoS ONE. 2019;14(5):e0217005.

40. Kell DB, Pretorius E. Proteins behaving badly. Substoichiometric molecular control and amplification of the initiation and nature of amyloid fibril formation: lessons from and for blood clotting. Prog Biophys Mol Biol. 2017;123:16-41.

41. Page MJ, Thomson GJA, Nunes JM, Engelbrecht AM, Nell TA, de Villiers WJS, de Beer MC, Engelbrecht L, Kell DB, Pretorius E. Serum amyloid A binds to fibrin(ogen), promoting fibrin amyloid formation. Sci Rep. 2019:9(1):3102.

42. Pretorius E, Mbotwe S, Kell DB. Lipopolysaccharide-binding protein (LBP) reverses the amyloid state of fibrin seen in plasma of type 2 diabetics with cardiovascular co-morbidities. Sci Rep. 2017:7(1):9680.

43. Pretorius E, Page MJ, Engelbrecht L, Ellis GC, Kell DB. Substantial fibrin amyloidogenesis in type 2 diabetes assessed using amyloid-selective fluorescent stains. Cardiovasc Diabetol. 2017;16(1):141.

44. https://assets.thermofisher.com/TFS-Assets/CMD/manuals/Man-48204103-UltiMate-3000-RSLCnano-Man48204103-EN.pdf. Accessed 30 June 2021.

45. Zinellu A, Paliogiannis P, Carru C, Mangoni AA. Serum amyloid A concentrations, COVID-19 severity and mortality: an updated systematic review and meta-analysis. Int J Infect Dis. 2021;105:668-74.

46. Sack GH Jr. Serum amyloid A—a review. Mol Med. 2018;24(1):46.

47. Cheng L, Yang JZ, Bai WH, Li ZY, Sun LF, Yan JJ, Zhou CL, Tang BP. Prognostic value of serum amyloid $A$ in patients with COVID-19. Infection. 2020:48(5):715-22.

48. Li H, Xiang X, Ren $H$, Xu L, Zhao L, Chen X, Long H, Wang Q, Wu Q. Serum Amyloid $\mathrm{A}$ is a biomarker of severe Coronavirus Disease and poor prognosis. J Infect. 2020;80(6):646-55.

49. Randeria SN, Thomson GJA, Nell TA, Roberts T, Pretorius E. Inflammatory cytokines in type 2 diabetes mellitus as facilitators of hypercoagulation and abnormal clot formation. Cardiovasc Diabetol. 2019;18(1):72.

50. Kojouharova M. Classical complement pathway component C1 q: purification of human C1q, isolation of C1q collagen-like and globular head fragments and production of recombinant C1q-derivatives. Functional characterization. Methods Mol Biol. 2014;1100:25-42.

51. Ackermann M, Verleden SE, Kuehnel M, Haverich A, Welte T, Laenger F, Vanstapel A, Werlein C, Stark H, Tzankov A, et al. Pulmonary vascular endothelialitis, thrombosis, and angiogenesis in Covid-19. N Engl J Med. 2020;383(2):120-8.

52. Iba T, Levy JH, Levi M, Thachil J. Coagulopathy in COVID-19. J Thromb Haemost. 2020;18(9):2103-9. 
53. Zhang J, Tecson KM, McCullough PA. Endothelial dysfunction contributes to COVID-19-associated vascular inflammation and coagulopathy. Rev Cardiovasc Med. 2020;21(3):315-9.

54. Goshua G, Pine AB, Meizlish ML, Chang $\mathrm{CH}$, Zhang H, Bahel P, Baluha A, Bar N, Bona RD, Burns AJ, et al. Endotheliopathy in COVID-19-associated coagulopathy: evidence from a single-centre, cross-sectional study. Lancet Haematol. 2020;7(8):e575-82.

55. Wool GD, Miller JL. The impact of COVID-19 disease on platelets and coagulation. Pathobiology. 2021:88(1):15-27.

56. Medcalf RL, Keragala CB, Myles PS. Fibrinolysis and COVID-19: a plasmin paradox. J Thromb Haemost. 2020;18(9):2118-22.

57. Bouck EG, Denorme F, Holle LA, Middelton EA, Blair AM, de Laat B, Schiffman JD, Yost CC, Rondina MT, Wolberg AS, et al. COVID-19 and sepsis are associated with different abnormalities in plasma procoagulant and fibrinolytic activity. Arterioscler Thromb Vasc Biol. 2021;41(1):401-14.

58. Ajjan RA, Schroeder V. Role of complement in diabetes. Mol Immunol. 2019;114:270-7.

59. Ladikou EE, Sivaloganathan H, Milne KM, Arter WE, Ramasamy R, Saad R, Stoneham SM, Philips B, Eziefula AC, Chevassut T. Von Willebrand factor (VWF): marker of endothelial damage and thrombotic risk in COVID-19? Clin Med (Lond). 2020;20(5):e178-82.

60. Chen J, López JA. Interactions of platelets with subendothelium and endothelium. Microcirculation. 2005;12(3):235-46.

61. Feingold KR. Lipid and Lipoprotein Levels in Patients with COVID-19 Infections. In: Feingold KR, Anawalt B, Boyce A, Chrousos G, de Herder WW, Dhatariya K, Dungan K, Grossman A, Hershman JM, Hofland J et al, editors. Endotext. South Dartmouth (MA): MDText.com, Inc. Copyright @ 2000-2021, MDText.com, Inc.; 2020.

62. Hu X, Chen D, Wu L, He G, Ye W. Declined serum high density lipoprotein cholesterol is associated with the severity of COVID-19 infection. Clin Chim Acta. 2020:510:105-10.

63. Draxler DF, Sashindranath M, Medcalf RL. Plasmin: a modulator of immune function. Semin Thromb Hemost. 2017;43(2):143-53.

64. Cesarman-Maus G, Hajjar KA. Molecular mechanisms of fibrinolysis. Br J Haematol. 2005;129(3):307-21.
65. Kwaan HC, Lindholm PF. The central role of fibrinolytic response in COVID-19-a hematologist's perspective. Int J Mol Sci. 2021;22(3):1283.

66. Singh S, Saleem S, Reed GL. Alpha2-antiplasmin: the devil you don't know in cerebrovascular and cardiovascular disease. Front Cardiovasc Med. 2020;7:608899.

67. Reed GL, Houng AK, Singh S, Wang D. a2-antiplasmin: new insights and opportunities for ischemic stroke. Semin Thromb Hemost. 2017:43(2):191-9.

68. Seheult JN, Seshadri A, Neal MD. Fibrinolysis shutdown and thrombosis in severe COVID-19. J Am Coll Surg. 2020;231(2):203-4.

69. Reed GL, Matsueda GR, Haber E. Fibrin-fibrin and alpha 2-antiplasminfibrin cross-linking by platelet factor XIII increases the resistance of platelet clots to fibrinolysis. Trans Assoc Am Physicians. 1991;104:21-8.

70. Fraser SR, Booth NA, Mutch NJ. The antifibrinolytic function of factor XIII is exclusively expressed through $\mathrm{a}_{2}$-antiplasmin cross-linking. Blood. 2011;117(23):6371-4

71. Kell DB, Pretorius E. The simultaneous occurrence of both hypercoagulability and hypofibrinolysis in blood and serum during systemic inflammation, and the roles of iron and fibrin(ogen). Integr Biol (Camb). $2015 ; 7(1): 24-52$.

72. de Waal GM, de Villiers WJ, Pretorius E. The link between bacterial inflammagens, leaky gut syndrome and colorectal cancer. Curr Med Chem. 2021. https://doi.org/10.2174/0929867328666210219142737.

73. Pretorius L, Thomson GJA, Adams RCM, Nell TA, Laubscher WA, Pretorius E. Platelet activity and hypercoagulation in type 2 diabetes. Cardiovasc Diabetol. 2018;17(1):141.

74. Thachil J, Juffermans NP, Ranucci M, Connors JM, Warkentin TE, Ortel TL, Levi M, Iba T, Levy JH. ISTH DIC subcommittee communication on anticoagulation in COVID-19. J Thromb Haemost. 2020;18(9):2138-44.

\section{Publisher's Note}

Springer Nature remains neutral with regard to jurisdictional claims in published maps and institutional affiliations.
Ready to submit your research? Choose BMC and benefit from:

- fast, convenient online submission

- thorough peer review by experienced researchers in your field

- rapid publication on acceptance

- support for research data, including large and complex data types

- gold Open Access which fosters wider collaboration and increased citations

- maximum visibility for your research: over $100 \mathrm{M}$ website views per year

At $\mathrm{BMC}$, research is always in progress.

Learn more biomedcentral.com/submissions 